\title{
Zoo-housed female chimpanzee adopts local female-specific tradition upon immigrating into a new group
}

\author{
Zoë Goldsborough $^{\mathrm{a}, \mathrm{b}, \mathrm{c}, *}$, Christine E. Webb ${ }^{\mathrm{a}, \mathrm{d}}$, Frans B.M. de Waal ${ }^{\mathrm{a}, \mathrm{e}}$ and \\ Edwin J.C. van Leeuwen ${ }^{\text {f,g }}$ \\ ${ }^{a}$ Animal Ecology, Department of Biology, Utrecht University, Utrecht, The Netherlands \\ ${ }^{\mathrm{b}}$ Department for the Ecology of Animal Societies, Max Planck Institute for \\ Animal Behaviour, Konstanz, Germany \\ ${ }^{c}$ Department of Biology, University of Konstanz, Konstanz, Germany \\ ${ }^{d}$ Department of Human Evolutionary Biology, Harvard University, Cambridge, MA, USA \\ ${ }^{\mathrm{e}}$ Department of Psychology, Emory University, Atlanta, GA, USA \\ ${ }^{\mathrm{f}}$ Behavioral Ecology and Ecophysiology Group, Department of Biology, \\ University of Antwerp, Wilrijk, Belgium \\ ${ }^{g}$ Centre for Research and Conservation, Royal Zoological Society of Antwerp, \\ Antwerp, Belgium \\ *Corresponding author's e-mail address: zgoldsborough@outlook.com
}

Received 17 September 2020; initial decision 16 November 2020; revised 18 December 2020; accepted 9 February 2021; published online 25 February 2021

\begin{abstract}
Wild female chimpanzees typically migrate to a neighbouring community at the onset of sexual maturity, a process that can be dangerous and unpredictable. To mitigate the risk of rejection in the new community, immigrants may employ several behavioural strategies. During the integration of two chimpanzee females at Royal Burgers' Zoo (Arnhem, The Netherlands) one of the immigrant females rapidly copied a local tradition — the crossed-arm walk — which has been present in the group for over 20 years. She copied the behaviour after meeting only one resident female, and showed the behaviour frequently throughout a 6-month observation period following the introduction. The other immigrant female never adopted the crossed-arm walk, highlighting the variation in behaviour by immigrants upon integration, as well as the potential associated consequences: in a separate observation period 2 years later, the female who copied the local tradition appeared more socially integrated than the other immigrant female.
\end{abstract}




\section{Keywords}

chimpanzees, social integration, social learning, behavioural copying, tradition.

\section{Introduction}

Chimpanzees are a patrilocal species in which the females migrate to neighbouring communities upon becoming sexually mature (Goodall, 1986; Nishida et al., 1999). Such transitions are typically accompanied by social tension induced by the unpredictability of the resident chimpanzees, and it has been suggested that the migrating females may employ different strategies to cope with this tension (Luncz \& Boesch, 2014). In captivity, chimpanzee introductions are particularly difficult, stressful, and even dangerous for the individuals involved (Brent et al., 1997). One of the challenges for migrating females lies in acquiring knowledge of the local social dynamics (e.g., who is the dominant individual and which individuals might be positively inclined toward social interactions?). Social learning can be an efficient means to obtain such locally adaptive behaviour (Galef, 1995; Laland, 2004). Instead of sampling the local contingencies with risky and time-consuming trial-and-error learning, an individual may simply attend to the behaviour of residents and adopt their choices accordingly (Hoppitt \& Laland, 2013). This logic plausibly holds for tangible manipulations of the environment (e.g., what plants to eat, what material to use for extractive foraging or nest building), but what about social conventions of which the function is opaque or even non-existent apart from the fact that everybody's doing it, like the finger-in-mouth games in capuchin monkeys or the grass-in-ear behaviour in chimpanzees (Perry et al., 2003; McGrew, 2004; van Leeuwen et al., 2014)?

The matching of behaviour has been interpreted in terms of homophily: the principle that 'similarity breeds connection' (McPherson et al., 2001). Homophily in the form of preferred associations and interactions with conspecifics similar in phenotype has been shown in a variety of species (e.g., zebras (Sundaresan et al., 2007); dolphins (Lusseau \& Newman, 2004); meerkats (Madden et al., 2011); chimpanzees (Massen \& Koski, 2014)). For humans, it has even been shown that behavioural copying occurs unconsciously, with the underlying motivation assumed to be avoiding ostracism by increasing favourability (Lakin et al., 2008). 
Here, we report a case in which a captive immigrant female chimpanzee rapidly adopts a local female-specific tradition upon integrating into her new group. Such behavioural copying of idiosyncratic/arbitrary behaviour is reminiscent of chimpanzees imitating the wearing of grass in one own's ear (van Leeuwen et al., 2014), copying the posture and gait of a disabled group member (Hobaiter \& Byrne, 2010), and matching their food grunt utterances to those of the resident chimpanzees (Watson et al., 2015). Comparable arbitrary traditions have been observed in chimpanzees' closest living relative, the bonobo (Pan paniscus; de Waal, 1988; van Leeuwen et al., 2020). However, contrary to these studies, in the current observations, the behavioural copying occurred very quickly after "exposure" to a performer (within two days), and it was an immigrating individual who adopted the behaviour of residents, rather than a behaviour spreading in an already established group.

The behaviour under consideration is an idiosyncratic behaviour henceforth referred to as 'crossed-arm walk' - which has not previously been mentioned in chimpanzee ethograms (Nishida et al., 2010). The crossed-arm walk is defined as an individual walking quadrupedally while holding one arm crossed in front of their body, oftentimes emptyhandedly, but occasionally with food or a stick in the hand (see Figure 1 and the video at 10.6084/m9.figshare.13899740). Interestingly, the behaviour is exclusively performed by female members of the Royal Burgers' Zoo group. The immigrant female under consideration (Moni) was integrated into the group together with another immigrant female (Erika), who did not adopt the crossed-arm walk. By comparing their social integration thereafter, we explore whether behavioural copying might be an effective strategy for immigrating chimpanzee females to facilitate social acceptance. First, we detail Moni and Erika's respective backgrounds and introduction into the Royal Burgers' Zoo group. Second, we describe their behavioural strategies during the first months of the introduction with a specific focus on the crossed-arm walk. Lastly, we assess their social embeddedness in the new group across two temporally distinct study periods as a marker of social integration.

\section{Methods}

\subsection{Subjects and housing}

Subjects were 15 adult chimpanzees (4 males) housed at Royal Burgers' Zoo (the Netherlands); further subject demographics are available in Table A1 in 
A)

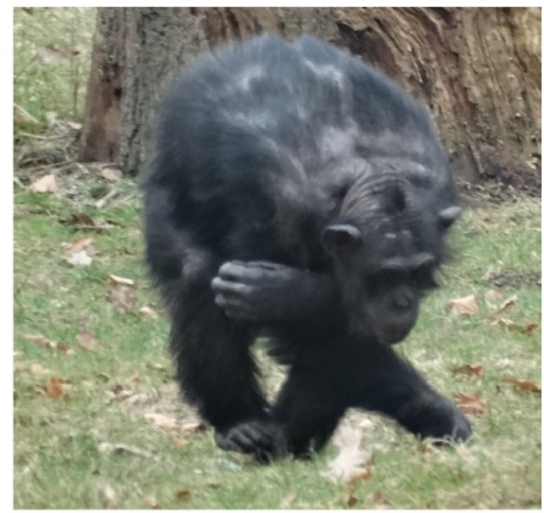

B)

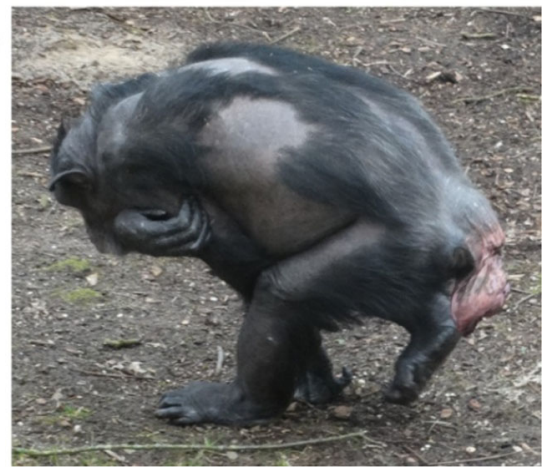

Figure 1. Pictures showing (A) a front view and (B) a side view of an individual engaging in the crossed-arm walk.

the Appendix. The chimpanzees had access to an indoor $\left( \pm 386 \mathrm{~m}^{2}\right)$ and outdoor enclosure $\left( \pm 7000 \mathrm{~m}^{2}\right)$, although sometimes they were restricted to one of the two. Two of the females in the group, Erika and Moni, arrived at the zoo on 18 November 2015 and were integrated on 25 October 2017 after an introduction process of 2 years (see Table A2 in the Appendix for a timeline and more detailed description). Moni was hand-reared and arrived at the zoo by herself after being housed solitary for several years. Erika was mother-reared and arrived together with her mother Marlene, with whom she was subsequently integrated. Marlene passed away due to heart failure on 14 April 2017. During the introduction the caretakers maintained extensive records consisting of daily notes and ad lib observations in a logbook, as well as video recordings of important events; however, these records were too unsystematic for formal analysis. 


\subsection{Data collection}

Data on affiliative, abnormal and idiosyncratic behaviour (including crossedarm walking) were collected from 20 November 2017 until 1 June 2018 (hereafter Period 1) by means of continuous focal observations (10 min/individual) by a pair of observers (one observing and one entering observations). Additionally, grooming interactions were sampled for $90 \mathrm{~min} /$ day with global (i.e., group-wide) scans of proximity $(<1 \mathrm{~m})$ every $10 \mathrm{~min}$. We collected an average of $\pm 14 \mathrm{~h}$ of focal data per individual, and $\pm 119 \mathrm{~h}$ of global data containing \pm 805 scans. Furthermore, from 24 January 2020 until 12 March 2020 (hereafter Period 2), another $32 \mathrm{~h}$ of global data were collected by a single observer. The protocol involved 60-min observations of the entire group, during which grooming was recorded continuously and proximity $(<1 \mathrm{~m})$ at 15 -min scan intervals, resulting in 160 scans. Observations were balanced across time of day and in the case of two observers, across observers.

\subsection{The 'crossed-arm walk' at Royal Burgers' zoo}

According to the chimpanzee caretakers, the crossed-arm walk was first shown by either a female named Moniek (born 1977) or her daughter Morami (born 1987). Morami most likely engaged in the crossed-arm walk already in 1995, when the following note was made in the caretakers' diary: "Morami has been walking weirdly with her arm for a while, doesn't use it at all" (translated from Dutch). No mention was made of an injury in the months before or after this entry. At that time, Morami was 6 months pregnant, and gave birth in January the following year (1996). Both Morami and Moniek have been observed engaging in the crossed-arm walk for at least twenty years (personal communication with two chimpanzee caretakers with over 15 and 20 years of experience with the chimpanzees respectively). However, the two females do not always locomote in this way (video at 10.6084/ m9.figshare.13899740) and, to our knowledge, have experienced no related physical injury or ailment, indicating that they are not suffering from a particular locomotion deficit. Other females, but not males (ZG \& caretakers, pers.obs.), have been observed to engage in the crossed-arm walk as well (see Results).

The crossed-arm walk has no clear adaptive value, on the contrary, it slows down an individual considerably as they are only using three limbs (video at 10.6084/m9.figshare.13899740). Possibly, it contributes to conserving 
warmth when outside, although the crossed-arm walk has been observed at similar rates in both the indoor and outdoor enclosure (Paired samples $t$-test, $\left.t=1.165, p=0.274, \mu_{\text {in }}=0.40, \mu_{\text {out }}=0.25\right)$. The frequency of crossedarm walking was assessed using the data from Period 1 (closest to onset integration). Prior to arriving at the zoo, neither of the immigrant females was observed to engage in crossed-arm walking (EAZA database).

\subsection{Introduction of the two immigrant females}

During the integration process, introductions mostly occurred one-on-one or two-on-two, and the group was kept in subgroups of varying sizes. The first individual that Moni was introduced to was Morami, on 10 December 2015, which was the first day that they made visual contact. At the end of the day they were allowed in the same enclosure. Morami was also the first group member Erika was introduced to, on 16 December 2015, when she and her mother Marlene were introduced to the sub-group containing Moni and Morami.

\subsection{Determining social embeddedness of the immigrant females}

To assess the social integration of the immigrants in both time periods, a Composite Sociality Index (CSI) was calculated for each individual (for more details, see Appendix). The CSI score reflects to what extent an individual deviates from the median of the group on several affiliative measures combined, and as such provides a measure of social integration (Sapolsky et al., 1997; Silk et al., 2003; Webb et al., 2017). A CSI score of 1 indicates that an individual is just as integrated as the median of the group (i.e., $<1$ indicates less integration; $>1$ indicates more integration). Furthermore, we considered the affiliative partners of Moni, the adopter of the crossed-arm walk, by examining who she directed most grooming towards and received most grooming from. In Period 1 we used hourly rates of grooming, in Period 2 dichotomous occurrence of grooming per session (see Appendix).

\subsection{Data availability statement}

The code and datasets used for analyses are available via https://doi.org/10. 5281/zenodo.4359328 


\section{Results}

\subsection{Behavioural strategies during integration: the crossed-arm walk}

Two days after her introduction to Morami, on 12 December 2015, Moni was first observed to engage in the crossed-arm walk (caretaker records). On 14 January 2016, she was recorded crossed-arm walking on a video of the introduction, while also housed with Morami. Erika was neither observed to perform the crossed-arm walk during the introduction period (caretaker records), nor during any other time up to the date of writing this report (caretaker reports and pers.obs., Period 1 and 2).

During Period 1, after the introduction was complete, crossed-arm walking was observed in the group 381 times (Table 1 and Figure 2). Every female in the group, including Moni, engaged in the crossed-arm walk at least once, except for Erika.

\subsection{Social integration of the immigrant females}

In Period 1, Erika had a CSI score of 0.44, the lowest score of the group, and Moni a score of 0.84 (11th/15 individuals). In Period 2, Erika's CSI score was 0.52 , the second-lowest, and Moni's CSI score was 1.33 (5th; see Figure 3). For all three measures used to calculate the CSI (grooming given, received grooming, and proximity), Moni ranked higher in Period 2 than in Period 1. Erika ranked the same for grooming given and proximity in both periods, while grooming received increased slightly (see Table A3

Table 1.

Frequency of crossed-arm walk per individual in Period 1.

\begin{tabular}{lc}
\hline Individual & Crossed-arm walk \\
\hline Gaby & 2 \\
Geisha & 1 \\
Jimmie & 2 \\
Moni & 94 \\
Moniek & 80 \\
Morami & 167 \\
Raimee & 2 \\
Roosje & 12 \\
Tesua & 1 \\
Tushi & 20 \\
\hline
\end{tabular}




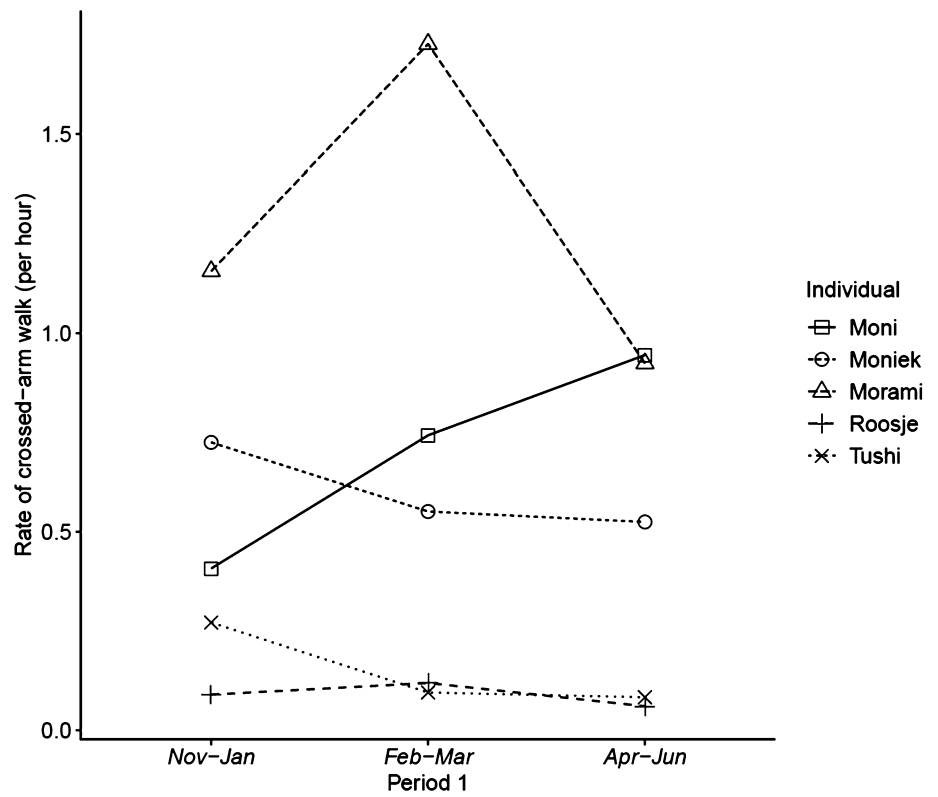

Figure 2. Rate of crossed-arm walk in Period 1 for all females for which frequency $>5$. The rates were calculated by dividing the frequency of crossed-arm walk by the time observed per female per month block (e.g., Nov-Jan).

in the Appendix for the rankings). The caretakers' records similarly noted Moni's improved position in the group over time, conveying that she interacted with more individuals and appeared less nervous, whereas no such improvement was noted for Erika. In Period 1, Moni directed most grooming towards Ghineau $(0.19 / \mathrm{h})$, followed by Moniek $(0.13 / \mathrm{h})$, Morami $(0.13 / \mathrm{h})$, and Tushi $(0.12 / \mathrm{h})$. She received most grooming from Tushi $(0.09 / \mathrm{h})$, followed by Giambo (0.04/h), Jimmie (0.04/h), and Jing (0.02/h). In Period 2, Moni directed most grooming towards Morami (occurring at least once in $25 \%$ of observation sessions), followed by Moniek (19\%), Gaby (12\%), and Geisha (9\%). She received most grooming from Geisha (6\%), followed by Ghineau (6\%), Raimee (6\%), and Tushi (6\%).

\section{Discussion}

In this report, we describe how an immigrant female chimpanzee (Moni) copies an idiosyncratic group-specific behaviour soon after being introduced to a new group. Two days after first contact with a resident female (a crossed- 


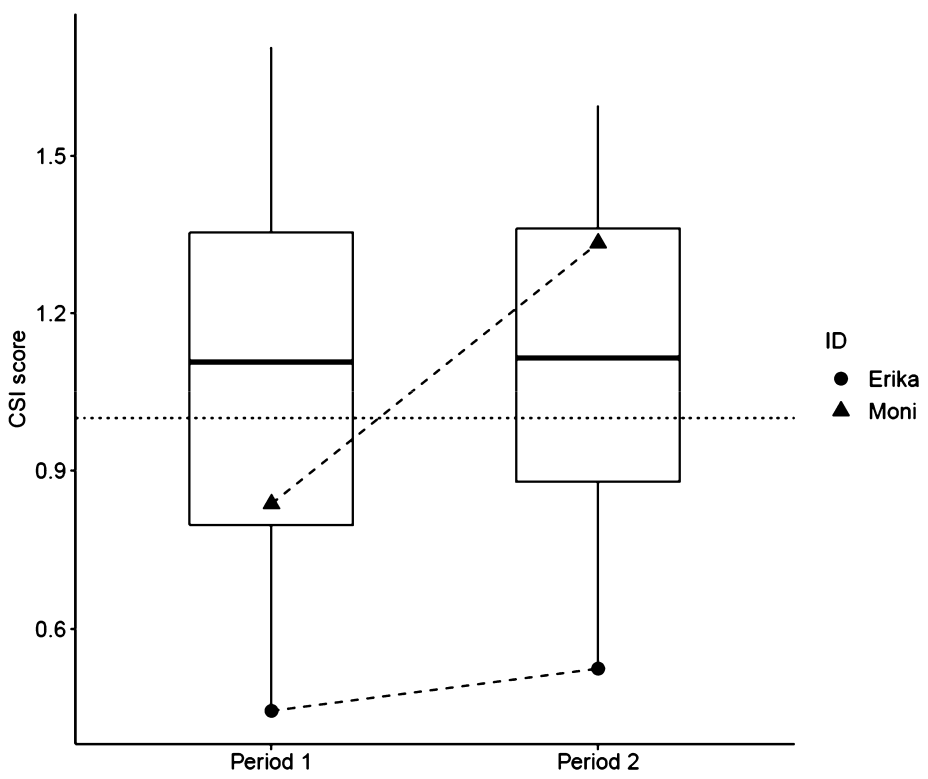

Figure 3. Boxplot of CSI scores in Period 1 and 2, including bold horizontal lines (medians), boxes (quartiles) and minimum and maximum (vertical lines). The CSI scores of Moni (triangle) and Erika (circle) are overlaid and connected by dotted lines.

arm walker), before meeting any other resident individuals, Moni engages in crossed-arm walking: an inefficient means of locomotion without clear function that is enacted by the majority of females in the new group. The immediacy of the acquisition of this behaviour by the immigrant, as well as the introduction context during which this behavioural copying occurred, both invite us to speculate that Moni may have enacted an integration strategy that facilitated social favourability (as suggested by Moni's steep increase in social integration over time). Another immigrant female (Erika) who similarly encountered the crossed-arm walkers first and for similar durations during integration - yet never adopted the crossed-arm walk — did not exhibit marked improvements in social integration over time. These findings provide anecdotal evidence that behavioural copying might be an expression of social motivation which in conjunction may facilitate social acceptance in chimpanzees.

Anecdotal evidence should be treated with care (Sarringhaus et al., 2005; Sándor \& Miklósi, 2020). Yet, it is noteworthy that the anecdote reported here does not stand on its own. There are at least two other reports showing 
that chimpanzees may copy behaviourally ineffective/functionless behaviour (Hobaiter \& Byrne, 2010; van Leeuwen et al., 2014) and some indications that wild female chimpanzees may gradually ( $>1$ year) adopt local conventions upon immigration (Luncz et al., 2012; Luncz \& Boesch, 2014). Hence, it is conceivable that Moni strategically adopted the local convention. Moni's behavioural copying upon immigration is further reminiscent of studies reporting conformity in migrating animals (Van de Waal et al., 2013; Luncz \& Boesch, 2014; Aplin et al., 2015). Especially when individuals are uncertain of their predicament, for instance during migrations, the inclination to align oneself with the (new) majority can take precedence (van Bergen et al., 2004; Morgan et al., 2012; Smolla et al., 2016; Jones et al., 2018). It is noteworthy that chimpanzee introductions in captive settings are notoriously difficult, often resulting in protracted aggression and injuries (Brent et al., 1997). This may further incentivize immigrating individuals to gain social acceptance from the group. In this light, it is relevant to emphasize that Erika, the immigrant who did not copy the crossed-arm walk, was introduced to the new group together with her mother, Marlene. As such, Erika might have been less uncertain/insecure in her new environment compared to Moni, who was introduced alone and had been housed solitary for years prior to her arrival. By extending her behavioural repertoire with the local customary behaviour of crossed-arm walking, Moni might have alleviated her uncertainty by gaining social benefits as expressed by her expedited social integration. The possibility that Moni engaged in behavioural copying in order to increase social favourability is consistent with the fact that Moni selectively directed her grooming efforts towards the two most prolific crossed-arm walkers (Morami and Moniek). Interestingly, though, while crossed-arm walk is shown by the majority of the Burgers' group, Moni first engaged in the behaviour after having met only one resident female. This suggests the workings of another yet related mechanism that could be sparked by uncertainty, namely copying the behaviour of the first individual one encounters (Galef \& Whiskin, 2008; van Leeuwen et al., 2016).

Similarly, given that close affiliative relationships are thought to facilitate social learning (Bonnie \& de Waal, 2006; Hobaiter et al., 2014; van Leeuwen et al., 2014; Lamon et al., 2017), it is also possible that Moni copied the crossed-arm walk from Morami given the nature of the social bonds between the females. According to the caretakers, Moni (but not Erika) and 
Morami responded very positively to one another when introduced, which is supported by the fact that they went from visual contact to sharing an enclosure within one day. However, as Moni engaged in the crossed-arm walk already two days after first meeting Morami, in our view, it is more plausible to infer that Moni proactively tried to establish connections by means of behavioural copying than the inverse alternative where Moni established close social bonds of which the behavioural copying was but an expression. This interpretation is corroborated by the observation that although Moni directed her grooming efforts selectively to Morami and Moniek, this directional social bonding behaviour was not selectively reciprocated. Interestingly, when an entire group of chimpanzees migrated to a resident group, the adoption of local behaviour by immigrant chimpanzees only occurred after affiliative social relationships had been formed (Watson et al., 2015). In conjunction, these findings suggest that chimpanzees may cope with immigration by converging toward locally adaptive information, but that the extent of experienced stress owing to uncertainty (higher when migrating alone like Moni - than with familiar group members — like Erika and in the study by Watson and colleagues) moderates its immediacy.

To our knowledge, the crossed-arm walk is the first female-specific tradition in chimpanzees ever reported. While sex-specific preferences in play object choices have been found in juvenile chimpanzees (Kahlenberg \& Wrangham, 2010), and sex differences in rates of social learning (Lonsdorf et al., 2004), there are no reports on sex-specific conventions in chimpanzees or primates in general. The crossed-arm walk tradition was presumably instigated by Morami, who was pregnant at the time. Given that the form of the crossed-arm walk (which closely resembles how a chimpanzee would carry an (deceased) infant), and the female-specific expression of the behaviour, it is tempting to speculate that maternal instincts could have played a role in the inception and spread of the behaviour. In this light, it is of interest to note that Erika is the only female in the group who has never had an infant.

The reported observations and inferences warrant differential scrutiny (Sándor \& Miklósi, 2020). Moni's adoption of the crossed-arm walk provides an objective account of chimpanzees' capacity to copy behavioural sequences (Hobaiter \& Byrne, 2010; van Leeuwen et al., 2014), yet proponents of the "zone of latent solutions" approach (Tennie et al., 2020) would challenge this notion by arguing that the crossed-arm walk was 
merely individually reinvented by Moni. In our view, however, the speed at which Moni adopted the crossed-arm walk, in conjunction with the idiosyncrasy of the crossed-arm walk posture/gait, defies — probabilistically — the reinvention hypothesis. The link between Moni's crossed-arm walk adoption and her facilitated social integration compared to the immigrant who did not copy the crossed-arm walk (Erika) is impossible to prove based on the reported observations. There are myriad reasons why Moni might have become more socially integrated than Erika - for instance, a difference in personality, a difference in life histories (i.e., Moni was hand-raised while Erika was mother-reared), and the differences in introduction contexts. Events during the observation period could also have affected differences in integration, for instance, Moni experienced a stillbirth during Period 1 and received increased affiliation from certain group members afterwards (Goldsborough et al., 2020). Therefore, our anecdotal report could best be interpreted as (i) evidence that chimpanzees can (and are motivated to) alter their behaviour upon integration into a new group (likely owing to social influences), and (ii) a tentative pointer to the possibility that in non-human animals behavioural similarity may induce relative favourability, just like in humans (e.g., McPherson et al., 2001). Tracking behavioural responses of animals integrating into new groups may thus represent an interesting approach for examining animals' phenotypic plasticity and exploring the evolutionary origins of behavioural copying and its ramifications for social acceptance.

\section{Acknowledgements}

We are grateful to Royal Burgers' Zoo for allowing this study to take place and to the chimpanzee caretakers for their dedication to the animals and assistance throughout the observations. EJCvL is supported in the capacity of postdoctoral fellow by the Research Foundation Flanders (FWO). CEW received a fellowship from the Living Links Center at Emory University which, together with Utrecht University, supported the research. We wish to thank Kayla Kolff for her assistance in collecting data for this study. Lastly, we wish to thank the chimpanzees, Erika, Fons, Gaby, Geisha, Ghineau, Giambo, Jimmie, Jing, Marlene, Moni, Moniek, Morami, Raimee, Roosje, Tesua and Tushi, without whom this research would not have been possible. 


\section{References}

Aplin, L.M., Farine, D.R., Morand-Ferron, J., Cockburn, A., Thornton, A. \& Sheldon, B.C. (2015). Experimentally induced innovations lead to persistent culture via conformity in wild birds. — Nature 518: 538-541. DOI:10.1038/nature13998.

Bonnie, K.E. \& de Waal, F.B.M. (2006). Affiliation promotes the transmission of a social custom: handclasp grooming among captive chimpanzees. — Primates 47: 27-34. DOI:10. 1007/s10329-005-0141-0.

Brent, L., Kessel, A.L. \& Barrera, H. (1997). Evaluation of introduction procedures in captive chimpanzees. —Zoo Biol. 16: 335-342. DOI:10.1002/(SICI)1098-2361(1997)16:4<335:: AID-ZOO5>3.0.CO;2-B.

de Waal, F.B.M. (1988). The communicative repertoire of captive bonobos (Pan Paniscus), compared to that of chimpanzees. - Behaviour 106: 183-251. DOI:10.1163/ $156853988 X 00269$.

Galef, B.G. (1995). Why behaviour patterns that animals learn socially are locally adaptive. — Anim. Behav. 49: 1325-1334. DOI:10.1006/anbe.1995.0164.

Galef, B.G. \& Whiskin, E.E. (2008). 'Conformity' in Norway rats? - Anim. Behav. 75: 2035-2039. DOI:10.1016/j.anbehav.2007.11.012.

Goldsborough, Z., van Leeuwen, E.J.C., Kolff, K.W.T., de Waal, F.B.M. \& Webb, C.E. (2020). Do chimpanzees (Pan troglodytes) console a bereaved mother? - Primates 61: 93-102. DOI:10.1007/s10329-019-00752-x.

Goodall, J. (1986). The chimpanzees of Gombe: patterns of behavior. - Belknap Press of Harvard University Press, Cambridge, MA.

Hobaiter, C. \& Byrne, R.W. (2010). Able-bodied wild chimpanzees imitate a motor procedure used by a disabled individual to overcome handicap. — PLoS ONE 5: e11959. DOI:10. 1371/journal.pone.0011959.

Hobaiter, C., Poisot, T., Zuberbühler, K., Hoppitt, W. \& Gruber, T. (2014). Social network analysis shows direct evidence for social transmission of tool use in wild chimpanzees. PLOS Biol. 12: e1001960. DOI:10.1371/journal.pbio.1001960.

Hoppitt, W. \& Laland, K. (2013). Social learning: an introduction to mechanisms, methods, and models. — Princeton University Press, Princeton, NJ. DOI:10.1515/9781400846504.

Jones, S., Czaczkes, T.J., Gallager, A.J. \& Bacon, J.P. (2018). Copy when uncertain: lower light levels result in higher trail pheromone deposition and stronger reliance on pheromone trails in the ant Lasius niger. — BioRxiv: 473579. DOI:10.1101/473579.

Kahlenberg, S.M. \& Wrangham, R.W. (2010). Sex differences in chimpanzees' use of sticks as play objects resemble those of children. — Curr. Biol. 20: R1067-R1068. DOI:10.1016/ j.cub.2010.11.024.

Lakin, J.L., Chartrand, T.L. \& Arkin, R.M. (2008). I am too just like you: nonconscious mimicry as an automatic behavioral response to social exclusion. - Psychol. Sci. 19: 816-822. DOI:10.1111/j.1467-9280.2008.02162.x.

Laland, K.N. (2004). Social learning strategies. — Anim. Learn. Behav. 32: 4-14. DOI:10. 3758/BF03196002. 
Lamon, N., Neumann, C., Gruber, T. \& Zuberbühler, K. (2017). Kin-based cultural transmission of tool use in wild chimpanzees. - Sci. Adv. 3: e1602750. DOI:10.1126/sciadv. 1602750.

Lonsdorf, E.V., Eberly, L.E. \& Pusey, A.E. (2004). Sex differences in learning in chimpanzees. — Nature 428: 715-716. DOI:10.1038/428715a.

Luncz, L.V. \& Boesch, C. (2014). Tradition over trend: neighboring chimpanzee communities maintain differences in cultural behavior despite frequent immigration of adult females. — Am. J. Primatol. 76: 649-657. DOI:10.1002/ajp.22259.

Luncz, L.V., Mundry, R. \& Boesch, C. (2012). Evidence for cultural differences between neighboring chimpanzee communities. — Curr. Biol. 22: 922-926. DOI:10.1016/j.cub. 2012.03.031.

Lusseau, D. \& Newman, M.E.J. (2004). Identifying the role that animals play in their social networks. — Proc. Roy. Soc. Lond. B: Biol. Sci. 271(Suppl. 6): S477-S481. DOI:10.1098/ rsbl.2004.0225.

Madden, J.R., Drewe, J.A., Pearce, G.P. \& Clutton-Brock, T.H. (2011). The social network structure of a wild meerkat population: 3. Position of individuals within networks. Behav. Ecol. Sociobiol. 65: 1857-1871. DOI:10.1007/s00265-011-1194-2.

Massen, J.J.M. \& Koski, S.E. (2014). Chimps of a feather sit together: chimpanzee friendships are based on homophily in personality. — Evol. Hum. Behav. 35: 1-8. DOI:10.1016/ j.evolhumbehav.2013.08.008.

McGrew, W.C. (2004). The cultured chimpanzee: reflections on cultural primatology. Cambridge University Press, Cambridge. DOI:10.1017/CBO9780511617355.

McPherson, M., Smith-Lovin, L. \& Cook, J.M. (2001). Birds of a feather: homophily in social networks. - Annu. Rev. Sociol. 27: 415-444. DOI:10.1146/annurev.soc.27.1.415.

Morgan, T.J.H., Rendell, L.E., Ehn, M., Hoppitt, W. \& Laland, K.N. (2012). The evolutionary basis of human social learning. — Proc. Roy. Soc. Lond. B: Biol. Sci. 279: 653-662. DOI:10.1098/rspb.2011.1172.

Nishida, T., Kano, T., Goodall, J., McGrew, W.C. \& Nakamura, M. (1999). Ethogram and ethnography of Mahale chimpanzees. - Anthropol. Sci. 107: 141-188. DOI:10.1537/ase. 107.141.

Nishida, T., Zamma, K., Matsusaka, T., Inaba, A. \& McGrew, W.C. (2010). Chimpanzee behavior in the wild: an audio-visual encyclopedia. — Springer Japan, Tokyo. DOI:10. 1007/978-4-431-53895-0.

Perry, S., Baker, M., Fedigan, L., Gros-Louis, J., Jack, K., MacKinnon, K.C., Manson, J.H., Panger, M., Pyle, K. \& Rose, L. (2003). Social conventions in wild white-faced capuchin monkeys: evidence for traditions in a Neotropical primate. — Curr. Anthropol. 44: 241268. DOI: $10.1086 / 345825$.

Sándor, K. \& Miklósi, Á. (2020). How to report anecdotal observations? A new approach based on a lesson from "puffin tool use". — Front. Psychol. 11: 555487. DOI:10.3389/ fpsyg.2020.555487.

Sapolsky, R.M., Alberts, S.C. \& Altmann, J. (1997). Hypercortisolism associated with social subordinance or social isolation among wild baboons. — Arch. Gen. Psychiatr. 54: 11371143. DOI:10.1001/archpsyc.1997.01830240097014. 
Sarringhaus, L.A., McGrew, W.C. \& Marchant, L. (2005). Misuse of anecdotes in primatology: lessons from citation analysis. — Am. J. Primatol. 65: 283-288. DOI:10.1002/ajp. 20115.

Silk, J.B., Alberts, S.C. \& Altmann, J. (2003). Social bonds of female baboons enhance infant survival. - Science 302: 1231-1234. DOI:10.1126/science.1088580.

Smolla, M., Alem, S., Chittka, L. \& Shultz, S. (2016). Copy-when-uncertain: bumblebees rely on social information when rewards are highly variable. — Biol. Lett. 12: 20160188. DOI:10.1098/rsbl.2016.0188.

Sundaresan, S.R., Fischhoff, I.R., Dushoff, J. \& Rubenstein, D.I. (2007). Network metrics reveal differences in social organization between two fission-fusion species, Grevy's zebra and onager. — Oecologia 151: 140-149. DOI:10.1007/s00442-006-0553-6.

Tennie, C., Bandini, E., van Schaik, C.P. \& Hopper, L.M. (2020). The zone of latent solutions and its relevance to understanding ape cultures. - Biol. Philos. 35: 55. DOI:10.1007/ s10539-020-09769-9.

van Bergen, Y., Coolen, I. \& Laland, K.N. (2004). Nine-spined sticklebacks exploit the most reliable source when public and private information conflict. — Proc. Roy. Soc. Lond. B: Biol. Sci. 271: 957-962.

van de Waal, E., Borgeaud, C. \& Whiten, A. (2013). Potent social learning and conformity shape a wild primate's foraging decisions. - Science 340: 483-485. DOI:10.1126/ science. 1232769.

van Leeuwen, E.J.C., Cronin, K.A. \& Haun, D.B.M. (2014). A group-specific arbitrary tradition in chimpanzees (Pan troglodytes). - Anim. Cogn. 17: 1421-1425. DOI:10.1007/ s10071-014-0766-8.

van Leeuwen, E.J.C., Acerbi, A., Kendal, R.L., Tennie, C. \& Haun, D.B.M. (2016). A reappreciation of 'conformity'. — Anim. Behav. 122: e5-e10. DOI:10.1016/j.anbehav.2016. 09.010 .

van Leeuwen, E.J.C., Staes, N., Verspeek, J., Hoppitt, W.J.E. \& Stevens, J.M.G. (2020). Social culture in bonobos. - Curr. Biol. 30: R261-R262. DOI:10.1016/j.cub.2020.02. 038.

Watson, S.K., Townsend, S.W., Schel, A.M., Wilke, C., Wallace, E.K., Cheng, L., West, V. \& Slocombe, K.E. (2015). Vocal learning in the functionally referential food grunts of chimpanzees. — Curr. Biol. 25: 495-499. DOI:10.1016/j.cub.2014.12.032.

Webb, C.E., Romero, T., Franks, B. \& de Waal, F.B.M. (2017). Long-term consistency in chimpanzee consolation behaviour reflects empathetic personalities. - Nature Commun. 8: 292. DOI:10.1038/s41467-017-00360-7.

\section{Appendix}

\section{A1. Introduction process}

The first introductions of individuals always occurred 1 on 1 or 2 on 2 . The usual procedure was to place individuals in adjacent cages, where first they 
Table A1.

Subject demographics.

\begin{tabular}{lccl}
\hline Individual & Sex & Birth year & Maternal kinship \\
\hline Erika $^{*}$ & F & 1992 & Marlene (mother) \\
Fons & M & 1975 & \\
Gaby & F & 1984 & Ghineau (son) \\
Geisha & F & 1993 & \\
Ghineau & G & 2005 & \\
Giambo & G & 1989 & Jing (son) \\
Jimmie & F & 1960 & Jimmie (mother) \\
Jing & M & 1981 & Erika (daughter) \\
Marlene & F & 1975 & \\
Moni & F & 1989 & Morami (daughter) \\
Moniek & F & 1987 & Moniek (mother) \\
Morami & F & 1987 & Roosje (mother) \\
Raimee & F & 1999 & Raimee (daughter) \\
Roosje & F & 1979 & Tushi (full sibling) \\
Tesua & F & 1986 & Tesua (full sibling) \\
Tushi & F & 1992 & \\
\hline
\end{tabular}

* Introduced females.

${ }^{\text {a }}$ Passed away during introduction process on 14 March 2017.

could only have visual contact, then touching through a mesh or gap, and eventually full contact. If any of the individuals involved responded negatively, the introduction process did not continue to the next step (e.g. from visual to touching) and was tried again at a later moment. In the timeline above we highlight the most important events in the introduction, which is mostly limited to the formation of subgroups, and the introduction of the crossed-arm walkers to the new females. Relevant for our study is that once a sub-group was formed (for instance the female sub group of February 2016) individuals would briefly be taken out for 1 on 1 or 2 on 2 introductions, but this was never more than a few days and they always returned to their subgroup. Individuals from the "established" group were added to the "introduced" subgroup one by one following positive introductions with the new females, until the subgroups were fully merged. During the entire introduction process, the subgroups did have both visual and auditory contact with one another, but these were brief moments such as when switching between enclosures, and never prolonged visual contact or physical interaction. 
Table A2.

Timeline of introduction and relevant events, adapted from Webb et al. (2020) and supplemented with events related to the crossed-arm walk.

\begin{tabular}{|c|c|}
\hline Date & Event \\
\hline 11 November 2015 & $\begin{array}{l}\text { Moni/Erika/Marlene arrive at the zoo, are kept separate from the } \\
\text { group }\end{array}$ \\
\hline 10 December 2015 & Moni is introduced to Morami ${ }^{*}$ \\
\hline 12 December 2015 & Moni is noted as crossed-arm walking in the keeper records \\
\hline 16 December 2015 & $\overline{\text { Erika }}$ and Marlene are introduced to Moni and Morami ${ }^{*}$ \\
\hline 14 January 2016 & Moni recorded crossed-arm walking on video \\
\hline February 2016 & $\begin{array}{l}\text { Beginning of female "group" introduction } \\
\text { New group formed: Erika, Marlene, Moni, Morami*, Gaby, Geisha, } \\
\text { Tesua, Tushi } \\
\text { Still to introduce: Giambo, Jing, Fons, Ghineau, Jimmie, Moniek*, } \\
\text { Raimee, Roos }\end{array}$ \\
\hline July 2016 & $\begin{array}{l}\text { Semi-stable subgroups formed } \\
\text { Introduction: Erika, Marlene, Moni, Morami* }{ }^{*} \text {, Fons, Gaby, Ghineau, } \\
\text { Geisha, Tesua, Tushi } \\
\text { Established: Giambo, Jimmie, Jing, Moniek }{ }^{*} \text {, Raimee, Roosje }\end{array}$ \\
\hline 12 January 2017 & Moniek $^{*}$ added to introduction group \\
\hline 14 April 2017 & Marlene dies \\
\hline 25 October 2017 & Introduction complete, sub-groups merged \\
\hline
\end{tabular}

* The most active crossed-arm walkers.

\section{A2. Composite Sociality Index (CSI)}

In this research, to determine the composite sociality score (CSI), three affiliative measures were used. One measure was obtained from the scan samples: the proportion of scan points in proximity to another individual. The remaining measures, the hourly rate of grooming given and the hourly rate of grooming received, were obtained from the continuous recording in the global samples. To calculate the CSI, the following formula was used: $C S I=\left(\sum_{i=1}^{3} \frac{x_{i}}{m_{i}}\right) / 3$, where $x_{i}$ is the value of each affiliative measures and $m_{i}$ refers to the group median value per affiliative measure.

Owing to the fact that the absolute frequency of grooming interactions was determined differently in Period 1 compared to Period 2 (independent grooming bouts had to be separated by at least $30 \mathrm{~s}$ of non-grooming in Period 1, whereas in Period 2 even brief interruptions were regarded as boutends), we used a dichotomous measure of grooming per observation hour when calculating the 2020 CSI. 
Table A3.

Ranking of Moni and Erika per component of the CSI calculation in Period 1 and Period 2.

\begin{tabular}{lccccc}
\hline Behaviour from CSI & \multicolumn{2}{c}{ Ranking Moni (out of 15) } & & \multicolumn{2}{c}{ Ranking Erika (out of 15) } \\
\cline { 2 - 3 } \cline { 6 - 6 } & Period 1 & Period 2 & & Period 1 & Period 2 \\
\hline Grooming given & 7 th & 1 st & & 14 th & 14 th \\
Grooming received & 15 th & 12 th & & 14 th & 13 th \\
Proximity & 11 th & 7 th & & 15 th & 15 th \\
\hline
\end{tabular}

These rankings reflect how the scores of Moni and Erika on each component (e.g., the proportion of scan points in proximity) compare to those of the rest of the group.

Movie S1. Examples of crossed-arm walking. 